\title{
Most DC-SIGNR transcripts at mucosal HIV transmission sites are alternatively spliced isoforms
}

\author{
Huanliang Liu ${ }^{1,6,7}$, Florian Hladik ${ }^{2,6}$, Thomas Andrus ${ }^{1}$, Polachai Sakchalathorn ${ }^{2}$, \\ Gretchen M Lentz ${ }^{3}$, Michael F Fialkow ${ }^{3}$, Lawrence Corey ${ }^{1,2,4,5}$, M Juliana McElrath ${ }^{1,2,5}$ \\ and Tuofu $\mathrm{Zhu}^{*, 1,4}$
}

\begin{abstract}
${ }^{1}$ Department of Laboratory Medicine, University of Washington School of Medicine, Seattle, WA 98195, USA; ${ }^{2}$ Program in Infectious Diseases, Fred Hutchinson Cancer Research Center, Seattle, WA 98109, USA; ${ }^{3}$ Department of Obstetrics and Gynecology, University of Washington School of Medicine, Seattle, WA 98195, USA; ${ }^{4}$ Department of Microbiology, University of Washington School of Medicine, Seattle, WA 98195, USA; Department of Medicine, University of Washington School of Medicine, Seattle, WA 98195, USA
\end{abstract}

The repeat region of DC-SIGNR (CD209L) is polymorphic on the genomic level, and, in a separate study, we observed a correlation between the DC-SIGNR genotype and HIV-1 susceptibility during sexual contact. However, previous investigations using immunohistochemistry failed to detect membrane-bound DCSIGNR on cells in the genital and rectal mucosa. We therefore explored the presence of DC-SIGNR in these compartments with a more sensitive limiting dilution RT-PCR, which also allowed for quantification of alternatively spliced mRNA isoforms. DC-SIGN (CD209) and DC-SIGNR mRNA transcript isoforms were found in all 12 vaginal and two rectal biopsies obtained from 14 healthy individuals. For DC-SIGNR, we detected significantly more isoform than full-length transcripts (mean copy numbers/ $\mu \mathrm{g}$ RNA: 602 vs 26; $P=0.0009$ ). Four mucosal samples lacked full-length DC-SIGNR transcripts entirely. Cloning and sequencing of DC-SIGNR mRNA in three additional individuals revealed a diverse repertoire of DC-SIGNR isoforms, many of which encoded for proteins predicted to be soluble and secreted. Indeed, in one vaginal sample, we detected only soluble isoforms. In conjunction with our prior observation that the DC-SIGNR genotype has an effect on HIV-1 transmission in vivo, these findings emphasize that DC-SIGNR, in addition to DC-SIGN, should be considered as a cofactor in sexual HIV-1 transmission. Soluble isoforms, in particular, may modulate the efficiency of viral transmission and dissemination. European Journal of Human Genetics (2005) 13, 707-715. doi:10.1038/sj.ejhg.5201409 Published online 6 April 2005

Keywords: HIV-1; DC-SIGN (R); transcript isoforms

\section{Introduction}

Mucosal dendritic cells (DC) are believed to facilitate systemic dissemination of HIV-1 after sexual transmission.

*Correspondence: $\operatorname{Dr}$ T Zhu, Department of Laboratory Medicine, University of Washington School of Medicine, Box 358070, 1959 N.E. Pacific Street, Seattle, WA 98195-8070, USA.

Tel: + 1206732 6079; Fax: + 1206732 6109;

E-mail: tzhu@u.washington.edu

${ }^{6}$ These authors contributed equally to this work.

${ }^{7}$ Current address: Department of Microbiology and Immunology, University of Miami School of Medicine, Miami, FL 33136, USA

Received 13 July 2004; revised 16 February 2005; accepted 17 February 2005
This is accomplished by first sequestering viable virions through interaction with C-type lectins, most notably DCSIGN (CD209) (dendritic cell-specific intercellular adhesion molecule-3-grabbing nonintegrin), and then passing virus to $\mathrm{CD} 4{ }^{+}$T cells. ${ }^{1-4}$ DC-SIGN is abundantly expressed on many types of DCs including those derived in vitro from monocytes as well as DCs located in the genital stroma and within lymphoid tissues. ${ }^{5-9}$ DC-SIGN binds the HIV-1 surface glycoprotein 120 (gp120) with high affinity in a CD4-independent manner. ${ }^{1,10}$ A close homologue to DCSIGN, DC-SIGNR (DC-SIGN related) (CD209L), exhibits a similar capacity to bind HIV-1. ${ }^{11-13}$ Immunohistochemical 
staining revealed that expression of DC-SIGNR was distinct from DC-SIGN and occurred predominantly in the endothelia of the liver, lymph nodes, and placenta. ${ }^{11,12,14}$ In addition, some studies demonstrated mRNA encoding DCSIGNR also in monocyte-derived DCs, albeit at a significantly lower level than DC-SIGN mRNA. ${ }^{11,15,16}$

Both DC-SIGN and DC-SIGNR are organized into three domains: an N-terminal cytoplasmic domain, a repeat region containing seven repeats of a 23 amino-acid sequence, and a C-terminal domain with homology to C-type lectins. ${ }^{11,15}$ The repeat region of DC-SIGNR is polymorphic at the genomic level. Seven different alleles for the DC-SIGNR repeat region have been identified, encoding from three to nine repeats. ${ }^{11}$ In contrast, the repeat region of DC-SIGN is highly conserved. ${ }^{11,17}$ Both DC-SIGN and DC-SIGNR genes produce diverse isoform repertoires through alternative splicing of premRNA, resulting in membrane-bound and soluble proteins. ${ }^{16}$ These molecular variations could exert influence on the susceptibility to HIV-1 infection, as has been demonstrated for other HIV-1-binding receptors. ${ }^{18-20}$ This possibility is supported by our finding that homozygosity for the DCSIGNR 7-repeat allele (repeat region genotype 7/7) is associated with increased risk for HIV-1 infection, whereas the $7 / 5$ genotype seems to confer relative protection. ${ }^{21}$ Furthermore, one of our other studies showed that repeat region variations of DC-SIGN also affect HIV-1 transmission. ${ }^{17}$

Based on the association of certain DC-SIGNR genotypes with susceptibility to $\mathrm{HIV}-1$ infection in vivo, ${ }^{21}$ we hypothesized that alternative forms of DC-SIGNR are present in the genital and rectal mucosa, which exert influence on the acquisition of HIV-1 infection during sexual contact. Despite the lack of prior immunohistochemical evidence for DC-SIGNR in the mucosa, ${ }^{5}$ we speculated that if, for example, soluble isoforms dominated locally, immunohistochemistry may not have been a sufficiently sensitive detection technique. Therefore, we applied a more sensitive limiting dilution nested PCR to identify DC-SIGNR mRNA in vaginal and rectal biopsies, and estimated the contribution of DC-SIGNR isoforms to the entire pool of mucosal DC-SIGN and DC-SIGNR. We found both DC-SIGN and DC-SIGNR isoforms in all mucosal samples from 17 individuals. We also confirm that the extensive transcript repertoire of DC-SIGNR reported in a recent study ${ }^{16}$ extends to the vaginal and rectal mucosa. For DC-SIGNR, isoforms were much more frequent than full-length transcripts and often encoded proteins predicted to be soluble. Our results suggest that C-type lectin isoforms, in particular secreted products, may be potentially important factors in mucosal transmission of HIV-1.

\section{Materials and methods DC culture}

Blood was obtained from four healthy volunteers recruited at the Fred Hutchinson Cancer Research Center HIV
Vaccine Trials Unit (FHCRC HVTU). Mononuclear cells were isolated by Ficoll-Hypaque density centrifugation and enriched for DC precursors and monocytes by plastic adherence for $90 \mathrm{~min}$ at $37^{\circ} \mathrm{C}$ and $5 \% \quad \mathrm{CO}_{2} \cdot{ }^{22}$ The nonadherent fraction was discarded and the adherent cells were cultured with recombinant GM-CSF and IL-4 (PeproTech, Rocky Hill, NJ, USA) in RPMI 1640 supplemented with $100 \mathrm{U} / \mathrm{ml}$ penicillin, $100 \mu \mathrm{g} / \mathrm{ml}$ streptomycin, $1 \mathrm{mM}$ HEPES (BioWhittaker, Walkersville, MD, USA), and $10 \%$ heat-inactivated FBS (Gemini, Calibasas, CA, USA) (complete medium). The cytokines were added to the cultures at $800 \mathrm{U} / \mathrm{ml}$ on days 0,3 , and 6 . On day 6 , cultures were supplemented with monocyte-conditioned medium $(\mathrm{MCM})$ at $25 \%(\mathrm{v} / \mathrm{v})$ to induce maturation. ${ }^{23}$ Mature DCs were collected on day 7 .

\section{Collection of rectal and vaginal tissues}

Vaginal tissues were collected from 13 HIV-1 uninfected women undergoing vaginal repair surgery at the University of Washington and Swedish Medical Center in Seattle. Rectal biopsies (3-4 mm) were collected from four HIV-1 uninfected men recruited at the FHCRC HVTU. The specimens were transported to the laboratory within $2 \mathrm{~h}$ of procurement, washed in PBS, submerged in RNA stabilization reagent, and stored at $-20^{\circ} \mathrm{C}$.

\section{Genotyping of DC-SIGN and DC-SIGNR repeat region polymorphisms}

The DC-SIGN and DC-SIGNR repeat regions in exon 4 were amplified from genomic DNA, extracted from tissues or in vitro monocyte-differentiated DCs, with the following primer pairs: 1F1: 5'-CCACTTTAGGGCAGGAC-3' and 1R: 5'-AGCAAACTCACACCACACAA-3' (DC-SIGN); ${ }^{17}$ L28: 5'-TG TCCAAGGTCCCCAGCTCCC-3' and L32: 5'-GAACTCACC AAATGCAGTCTTCAAATC-3' (DC-SIGNR). ${ }^{11}$ PCR amplifications were performed in a volume of $30 \mu \mathrm{l}$ (DC-SIGN) or $50 \mu \mathrm{l}$ (DC-SIGNR) containing $0.25 \mathrm{mM}$ dNTPs, $1.0 \mu \mathrm{M}$ of each primer, $2.5 \mathrm{mM} \mathrm{MgCl}_{2}$ and $1.0 \mathrm{U}$ of Expand High Fidelity PCR System Enzyme in $1 \times$ reaction buffer (Roche, Indianapolis, IN, USA). The cycle conditions for DC-SIGN were $94^{\circ} \mathrm{C}$ for $5 \mathrm{~min}$, followed by 40 cycles of $95^{\circ} \mathrm{C}$ for $15 \mathrm{~s}$, $60^{\circ} \mathrm{C}$ for $30 \mathrm{~s}$, and $72^{\circ} \mathrm{C}$ for $30 \mathrm{~s}$, then followed by one cycle of $72^{\circ} \mathrm{C}$ for $7 \mathrm{~min} .{ }^{17}$ The cycle conditions for DC-SIGNR were modified from Bashirova et $a l^{11}$ and were $94^{\circ} \mathrm{C}$ for $5 \mathrm{~min}$, followed by 35 cycles of $94^{\circ} \mathrm{C}$ for $5 \mathrm{~s}$ and $70^{\circ} \mathrm{C}$ for $1 \mathrm{~min}$, then followed by incubation at $70^{\circ} \mathrm{C}$ for $7 \mathrm{~min}$. Alleles were distinguished by electrophoretic size fractionation of amplified products in 3\% agarose gel and staining with ethidium bromide.

\section{Detection of DC-SIGNR membrane and soluble mRNA isoforms}

Total RNA was extracted from DCs and mucosal biopsies by Qiagen RNeasy Protect Kit (Qiagen, Valencia, CA, USA). Fragments spanning the entire DC-SIGNR coding region 
were reverse transcribed (RT) and then amplified by nested PCR. The following primers were used: RT primer RR: 5'-GACGTTACAACATTTACCACTT- $3^{\prime} ;{ }^{15}$ first PCR primers RR and RF: 5'-AACATCTGGGGACAGCG-3'; second PCR primers RcR: 5'-AGGCTAGCAGGGAAACAACT-3' and RcF: 5'-TGGGGACAGCGGGAAAACAT-3'. RNA was RT in the presence of $1 \times$ first-strand buffer, $5 \mathrm{~mm}$ DTT, $200 \mathrm{U}$ SUPERSCRIPT II RNase $\mathrm{H}^{-}$Reverse Transcriptase (Invitrogen, Carlsbad, CA, USA), $2.0 \mathrm{~mm}$ dNTPs, and $2.5 \mu \mathrm{M}$ RT primer in a final reaction volume of $20 \mu \mathrm{l}$. Reverse transcription was carried out at $42^{\circ} \mathrm{C}$ for $50 \mathrm{~min}$, followed by a 15 -min step at $75^{\circ} \mathrm{C}$, and then cooling to $4^{\circ} \mathrm{C}$. In all, $2 \mu \mathrm{l}$ of the RT and first PCR products were used for first and second PCR, respectively. Both first and second PCR reactions were performed in a volume of $50 \mu \mathrm{l}$ containing $0.25 \mathrm{mM}$ dNTPs, $1.0 \mu \mathrm{M}$ of each primer, $2.5 \mathrm{mM} \mathrm{MgCl}_{2}$, and $1.5 \mathrm{U}$ of Expand High Fidelity PCR System Enzyme in $1 \times$ reaction buffer (Roche, Indianapolis, IN, USA). The first PCR conditions were $94^{\circ} \mathrm{C}$ for $3 \mathrm{~min}$, followed by 30 cycles for $94^{\circ} \mathrm{C}$ for $30 \mathrm{~s}, 55^{\circ} \mathrm{C}$ for $30 \mathrm{~s}$ and $72^{\circ} \mathrm{C}$ for $1 \mathrm{~min} 15 \mathrm{~s}$, then followed by incubation at $72^{\circ} \mathrm{C}$ for $7 \mathrm{~min}$. The second PCR conditions were $94^{\circ} \mathrm{C}$ for $3 \mathrm{~min}$, followed by 35 cycles for $94^{\circ} \mathrm{C}$ for $30 \mathrm{~s}, 56^{\circ} \mathrm{C}$ for $30 \mathrm{~s}$ and $72^{\circ} \mathrm{C}$ for $1 \mathrm{~min} 15 \mathrm{~s}$, then followed by incubation at $72^{\circ} \mathrm{C}$ for $7 \mathrm{~min}$. The PCR products were distinguished by $2 \%$ agarose gel electrophoresis with ethidium bromide staining. The major bands were excised, purified, cloned into the PCR3.1 vector (Invitrogen, Carlsbad, CA, USA) and sequenced on both strands using an ABI Big Dye Terminator Cycle Sequencing Kit (Applied Biosystems, Foster City, CA, USA).

\section{Measurement of DC-SIGN and DC-SIGNR mRNA copy numbers}

Total RNA extraction and reverse transcription of DC-SIGN and DC-SIGNR was performed as described above. For DCSIGN, the RT primer NR: 5'-CTAAAGGTCGAAGGATGGAG$3^{\prime}$ was used. Next, a limiting dilution nested $\mathrm{PCR}^{24,25}$ was performed, in which serial 5-fold dilutions of cDNA were prepared in triplicate for PCR. For the first and second PCR of DC-SIGNR, the same primer pairs as above were used. Nested PCR for DC-SIGN was performed with the first PCR primers NR and NF: 5'-CTAAAGCAGGAGTTCTGGAC-3'; and the second PCR primers DCC1: 5'-ACTGGGGGAGA GTGGGGTAC-3' and DCC2: 5'-AAAAGGGGGTGAAGTTC TGCT- $3^{\prime}$. The conditions for DC-SIGNR RT and nested PCR were the same as above, and identical conditions were also used for DC-SIGN RT and nested PCR. Four RNAse-free water controls were always run in parallel, two starting from the RT and two starting from the first PCR. Transcripts were distinguished by $2 \%$ agarose gel electrophoresis and ethidium bromide staining. Transcript lengths of $1255 \mathrm{bp}$ for DC-SIGN and 1223 bp for DC-SIGNR represented the 7repeat wild-type allele, and fragments of other lengths were considered transcript isoforms. Relative copy numbers of each transcript were determined as described previously using the computer program 'QUALITY'. ${ }^{26}$ The general assay variability for DC-SIGN and DC-SIGNR, full-length and isoform, mRNA copy number determination was estimated by repeating the complete limiting dilution RTPCR five times in two tissue donors. Fold-differences between the minimum and maximum copy numbers across each set of replicates ranged from 2.34 to 6.15 (mean 4.1). Copy numbers for full-length DC-SIGNR transcripts were consistently zero across the five repeat assays in a donor with the DC-SIGNR repeat region genotype $5 / 5$.

\section{Statistical analyses}

Copy numbers between DC-SIGN and DC-SIGNR isoform transcripts, as well as between full-length and isoform transcripts were compared using the Wilcoxon signed rank test. The proportion of individuals with at least one DCSIGNR 7-repeat allele was compared between individuals who lacked and individuals who possessed DC-SIGNR fulllength transcripts using Fisher's exact test. All tests were two-sided with a $P$-value of $<0.05$ considered significant. All analyses were performed using InStat Version 3.0 and PRISM version 4.0 (GraphPad Software, INC, San Diego, CA, USA).

\footnotetext{
Results Expression of DC-SIGN and DC-SIGNR transcripts in rectal and vaginal biopsies

Immunohistochemical studies have demonstrated DCSIGN expression on DCs infiltrating the genital and rectal mucosa, but failed to detect DC-SIGNR in these tissues. ${ }^{5,6}$ Nevertheless, our finding that certain DC-SIGNR genotypes correlate with the risk for HIV-1 infection ${ }^{21}$ may signify the presence of this molecule at the site of viral invasion. We therefore tested for vaginal and rectal expression of DCSIGNR using a more sensitive molecular technique, nested RT-PCR, and found DC-SIGNR mRNA in all 13 vaginal and four rectal biopsies tested (Tables 1 and 2). As predicted from the immunohistochemical analyses, ${ }^{5,6}$ nested RT-PCR also demonstrated the consistent presence of DC-SIGN mRNA when we analyzed 12 of the vaginal and two of the rectal biopsies (Table 2). For control purposes, we also analyzed DC-SIGN and DC-SIGNR mRNA expression by in vitro monocyte-derived DCs of four additional individuals. In all four DC samples, DC-SIGN (not shown) and DCSIGNR (Table 1) mRNA was present, which is in concordance with previous studies. ${ }^{11,15,16}$ Thus, these results corroborate the expression of DC-SIGN in vaginal and rectal mucosa. At the same time, we consistently found mRNA for DC-SIGNR in these tissues, indicating that this molecule is present as well, either in its membrane-bound or soluble form.
} 
Table 1 Heterogeneity of DC-SIGNR transcript isoforms from human rectal and vaginal mucosa, and monocytederived DCs

\begin{tabular}{|c|c|c|c|c|}
\hline $\begin{array}{l}\text { Sample } \\
\text { ID }\end{array}$ & $\begin{array}{l}\text { Repeat } \\
\text { region } \\
\text { genotype }\end{array}$ & Source & $\begin{array}{l}\text { Number } \\
\text { of repeats }\end{array}$ & Deletions $^{\mathrm{a}}$ \\
\hline 15 & $7 / 5$ & Vagina & $\begin{array}{l}5 \\
5 \\
0 \\
0 \\
0\end{array}$ & $\begin{array}{l}E \text { II, III, R5, } 6 \\
E \text { III, R5, } 6, E \text { VI } \rightarrow \text { new } \\
\text { C-terminus } \\
E \text { III, most of E IV to } \\
\text { part of } E V^{b} \\
\text { Part of } E \text { I to part of } E \\
V^{b} \\
\text { Part of } E \text { I to part of } E \\
V, E I^{b}\end{array}$ \\
\hline 16 & $7 / 5$ & $\mathrm{DC}$ & $\begin{array}{l}7 \\
6 \\
5 \\
4 \\
0\end{array}$ & $\begin{array}{l}\text { R6 } \\
\text { R5, } 6 \\
\text { R2-4 } \\
\text { Part of E II, III-VI, } \\
\text { retained intron I, } \\
\text { Part of intron } \mathrm{VI}^{\mathrm{b}}\end{array}$ \\
\hline 17 & $7 / 5$ & Rectum & $\begin{array}{l}7 \\
5 \\
4 \\
1 \\
1 \\
1 \\
1\end{array}$ & $\begin{array}{l}E \text { II, last } 69 \text { bp of } E \text { IV } \\
R 5,6 \\
R 4-6 \\
\text { most of } R 2 \text { to part of } E \\
V I^{b} \\
E \text { II, most of R2 to part } \\
\text { of E VI } \\
E \text { III, most of R2 to part } \\
\text { of } E \text { VI }\end{array}$ \\
\hline 18 & $7 / 7$ & $\mathrm{DC}$ & $\begin{array}{l}7 \\
7 \\
7 \\
5\end{array}$ & $\begin{array}{l}\text { E II } \\
\text { E II, VI } \rightarrow \text { new C- } \\
\text { terminus } \\
\text { E II, R4, 5, E VI } \rightarrow \text { new } \\
\text { C-terminus }\end{array}$ \\
\hline 19 & $7 / 7$ & $\mathrm{DC}$ & $\begin{array}{l}7 \\
7 \\
6 \\
5 \\
3\end{array}$ & $\begin{array}{l}\text { E II, III } \\
\text { E II, III, R1 } \\
\text { E II, R4, } 5 \\
\text { R3-6 }\end{array}$ \\
\hline 20 & $7 / 6$ & Rectum & $\begin{array}{l}7 \\
6 \\
6 \\
5 \\
4\end{array}$ & $\begin{array}{l}\text { E II, III } \\
\text { R5, E VI } \rightarrow \text { new C- } \\
\text { terminus } \\
\text { E II, R5 } \\
\text { R1, 5 } \\
\text { R2-4 }\end{array}$ \\
\hline 21 & $6 / 6$ & $\mathrm{DC}$ & $\begin{array}{l}6 \\
6 \\
6 \\
6 \\
5 \\
3 \\
2 \\
2\end{array}$ & $\begin{array}{l}\text { R5 } \\
\text { R5, last } 69 \text { bp of E IV } \\
\text { E III, R5 } \\
\text { E II, III, R1 } \\
\text { R2, 3 } \\
\text { E III, R2-5, other } 2 \text { bp }^{b} \\
\text { E II, III, R2-6 } \\
\text { E III, R2-6 }\end{array}$ \\
\hline
\end{tabular}

$D C$, monocyte-derived dendritic cells; $R$, repeat; $E$, exon.

${ }^{a}$ Roman numerals denote the seven exons of DC-SIGNR. $R$ in conjunction with an Arabic numbers denotes the seven full repeats of exon IV.

bEarly stop codon.

\section{DC-SIGN and DC-SIGNR genotypes}

Extensive genomic polymorphism of the DC-SIGNR repeat region has been reported ${ }^{11,21,27}$ and we sought to confirm this in our cohort. One individual exhibited five repeats on both alleles (5/5), two individuals had a $6 / 5$ genotype, one individual a 6/6, six individuals a 7/5, six individuals a 7/6, four individuals a 7/7 and one individual a 9/7 genotype (Tables 1 and 2). In contrast, we found only homozygous $7 /$ 7 repeat region genotypes for DC-SIGN (Table 2). These findings confirm the strong genomic polymorphism of the DC-SIGNR repeat region, ${ }^{11,21,27}$ which stands apart from the near complete homogeneity of the DC-SIGN repeat region. ${ }^{11,17}$

\section{DC-SIGNR transcript repertoire}

The genomic polymorphism of the DC-SIGNR repeat region, ${ }^{11,21,27}$ in conjunction with alternative splicing of DC-SIGNR pre-mRNA demonstrated in the placenta and liver, ${ }^{16}$ implies the potential for generating a wide variety of DC-SIGNR transcripts. Since we detected DC-SIGNR mRNA in all vaginal and rectal samples, we proceeded to investigate its actual transcript repertoire in these tissues. We purified and cloned the major DC-SIGNR RT-PCR products obtained from one vaginal, two rectal and four monocyte-derived DC samples (Figure 1 depicts selected bands for five of these samples), and then randomly

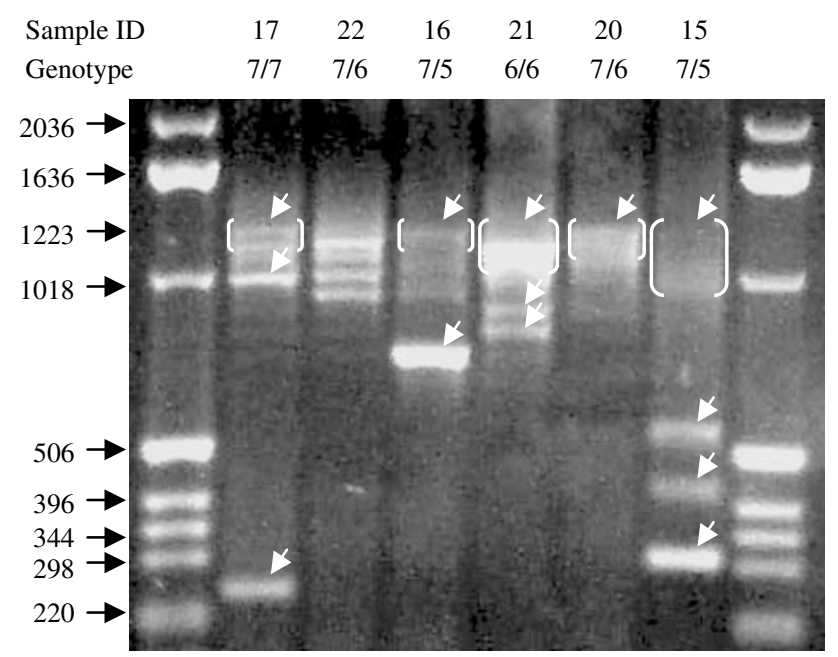

Figure 1 Detection and cloning of DC-SIGNR mRNA. Reverse transcription, nested PCR, and gel electrophoresis were performed as described in Materials and methods. A representative gel with five samples is shown. The full-length transcript length of DC-SIGNR is $1223 \mathrm{bp}$. Bands at other locations on the gel are transcript isoforms. The white arrows point to bands, or areas marked by parentheses, which were excised for cloning and sequencing. Samples 17, 22, 16, and 21 are monocyte-derived DCs, sample 20 is a rectal biopsy, and sample 15 is a vaginal biopsy. The individual sample identification numbers (ID) and DC-SIGNR repeat region genotypes are indicated on top, the base pair lengths to the left of the gel. Sample 22 has not been sequenced. 
Table 2 Relative DC-SIGN and DC-SIGNR full-length and isoform mRNA transcript levels in vaginal and rectal mucosa

\begin{tabular}{|c|c|c|c|c|c|c|c|c|c|}
\hline \multirow[b]{2}{*}{ Sample ID } & \multirow[b]{2}{*}{ Tissue } & \multicolumn{4}{|c|}{$D C-S I G N$} & \multicolumn{4}{|c|}{$D C-S I G N R$} \\
\hline & & $\begin{array}{l}\text { Repeat region } \\
\text { genotype }\end{array}$ & Full-length ${ }^{\mathrm{a}}$ & Isoform ${ }^{\mathrm{a}}$ & $\mathrm{F} / \mathrm{I}$ ratio $^{\mathrm{b}}$ & $\begin{array}{l}\text { Repeat region } \\
\text { genotype }\end{array}$ & Full-length ${ }^{\mathrm{a}}$ & Isoform ${ }^{\mathrm{a}}$ & $\mathrm{F} / \mathrm{I}$ ratio $^{\mathrm{b}}$ \\
\hline 1 & $\mathrm{~V}$ & $7 / 7$ & 900 & 361 & 2.49 & $5 / 5$ & 0 & 102 & 0 \\
\hline 2 & $\mathrm{~V}$ & $7 / 7$ & 3061 & 186 & 16.49 & $6 / 5$ & 0 & 24 & 0 \\
\hline 3 & V & $7 / 7$ & 16135 & 72 & 223.60 & $6 / 5$ & 0 & 310 & 0 \\
\hline 4 & $\mathrm{~V}$ & $7 / 7$ & 3627 & 68 & 53.29 & $7 / 5$ & 45 & 22 & 2.024 \\
\hline 5 & V & $7 / 7$ & 7850 & 134 & 58.45 & $7 / 5$ & 26 & 4811 & 0.005 \\
\hline 6 & V & $7 / 7$ & 12539 & 21 & 587.36 & $7 / 5$ & 16 & 28 & 0.580 \\
\hline 7 & $\mathrm{~V}$ & $7 / 7$ & 6468 & 248 & 26.12 & $7 / 6$ & 2.5 & 32 & 0.077 \\
\hline 8 & $\mathrm{~V}$ & $7 / 7$ & 2161 & 280 & 7.71 & $7 / 6$ & 4.7 & 134 & 0.035 \\
\hline 9 & V & $7 / 7$ & 32489 & 466 & 69.78 & $7 / 6$ & 11 & 42 & 0.271 \\
\hline 10 & V & $7 / 7$ & 15235 & 57 & 267.44 & $7 / 6$ & 5.7 & 14 & 0.406 \\
\hline 11 & $\mathrm{~V}$ & $7 / 7$ & 7224 & 88 & 82.13 & $7 / 7$ & 0 & 20 & 0 \\
\hline 12 & $\mathrm{~V}$ & $7 / 7$ & 1898 & 49 & 38.50 & $9 / 7$ & 4 & 46 & 0.087 \\
\hline 13 & $\operatorname{Re}$ & $7 / 7$ & 39734 & 1872 & 21.23 & $7 / 6$ & 14 & 107 & 0.129 \\
\hline 14 & $\mathrm{Re}$ & $7 / 7$ & 32999 & 1291 & 25.56 & $7 / 7$ & 242 & 2737 & 0.088 \\
\hline
\end{tabular}

${ }^{\mathrm{a}}$ Copies/ $\mu \mathrm{g}$ RNA.

${ }^{\mathrm{b}}$ Full-length/isoform ratio.

$\mathrm{V}$, vaginal biopsies; Re, rectal biopsies.

selected clones for sequencing. Per individual, four to eight distinctive isoforms were found, and in total, 31 distinct transcripts were identified (Table 1 and Figure 2). Of these, only one was the full-length transcript and 30 were isoforms containing previously unidentified deletions. ${ }^{16}$ Of these, 13 isoforms lacked exon III, which codes for the trans-membrane region, and were thus classified as soluble DC-SIGNR isoforms (Figure 2). In the vaginal sample, only these soluble isoforms were found. Early stop codons were introduced in eight isoforms, two were membrane-bound and six were soluble (Figure 2). New C termini with late stop codons were introduced in four isoforms, three were membrane-bound and one was soluble (Figure 2). Representative early and late stop codons are depicted in the alignment of predicted amino acid sequences of selected representative DC-SIGNR isoforms in Figure 3. Thus, genomic polymorphism and alternative splicing generates a large pool of DC-SIGNR transcript isoforms within a single individual and, even more so, on a population level. More importantly, this extensive transcript repertoire is not restricted to the placenta, liver, and lymph nodes, ${ }^{16}$ but is also expressed in the vaginal and rectal mucosa.

\section{Mucosal expression levels of DC-SIGN and DC-SIGNR full-length and isoform transcripts}

DC-SIGN expression in vaginal and rectal mucosa has been reported. ${ }^{1,5}$ Our finding that DC-SIGNR was also consistently transcribed in vaginal and rectal biopsies led us to compare the relative abundance of DC-SIGN with DCSIGNR transcripts in our mucosal samples. Since the transcript repertoire of DC-SIGNR in the mucosa appeared to be as extensive as described for the placenta, ${ }^{16}$ we extended our comparison to the relative contribution of full-length and isoform transcripts. A PCR-based limiting dilution strategy ${ }^{24,25}$ was used to quantify the relative copy numbers of DC-SIGN and DC-SIGNR mRNA full-length and isoform transcripts (Table 2). DC-SIGN full-length transcripts predominated (range 900-39734 copies, median 7537), whereas DC-SIGNR full-length transcripts were least abundant (range 0-242, median 4.4) (Figure 4). Isoform transcripts were present at intermediate levels (DC-SIGN: range 21-1872, median 80; DC-SIGNR: range 14-4811, median 37) and did not differ significantly from one another (Figure 4; $P=0.36$ ). DC-SIGNR isoform transcripts were much more abundant than DC-SIGNR full-length transcripts $(P=0.0009)$ (Figure 4$)$. In contrast, this relationship was inverted for DC-SIGN, where isoforms were less frequent than wild-type transcripts $(P=0.0001)$ (Figure 4). Of note, we detected only isoform and no fulllength transcripts for DC-SIGNR in four out of 14 mucosal biopsies (Table 2). Three of these four individuals completely lacked the DC-SIGNR 7-repeat allele at the genomic level, while all of the other 10 individuals possessed at least one 7-repeat allele ( $P=0.011$ for difference in proportions). Although the number of tested samples is low, these findings suggest that the 7-repeat allele is required for the generation of the full-length transcript. Taken together, DC-SIGN and DC-SIGNR isoform transcripts are consistently found in the genital and rectal mucosa at the site of HIV exposure. In the case of DC-SIGNR, these isoforms, many of which may represent soluble variants, predominate over the wild-type transcripts.

\section{Discussion}

DC-SIGNR is a homologue of DC-SIGN and exhibits a similar ability to bind HIV-1 gp120. ${ }^{11-13,15}$ However, its tissue distribution and cellular expression are not identical to DC-SIGN. ${ }^{1,5,11,12,14,15}$ Notably, immunohistochemical and flow cytometric analyses have failed to detect 


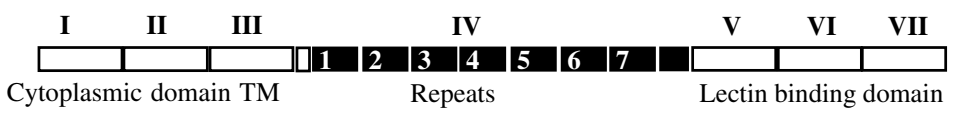

b

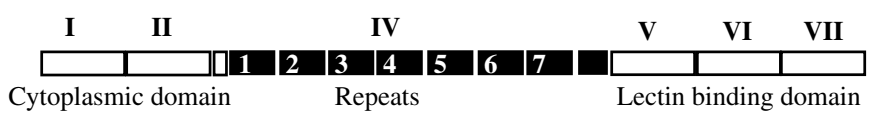

\section{c Membrane isoforms}

\begin{tabular}{|c|c|c|}
\hline Type & $\begin{array}{l}\text { No. of } \\
\text { repeats }\end{array}$ & Deletions \\
\hline 1 & 7 & \\
\hline 2 & 7 & E II \\
\hline 3 & 7 & E II, VI > new C terminus \\
\hline 4 & 7 & E II, last $69 \mathrm{bp}$ of E IV \\
\hline 5 & 6 & R5 \\
\hline 6 & 6 & R6 \\
\hline 7 & 6 & R5, E VI > new C terminus \\
\hline 8 & 6 & R5, last 69bp of E IV \\
\hline 9 & 6 & E II, R5 \\
\hline 10 & 5 & $\mathbf{R} 1,5$ \\
\hline 11 & 5 & $R 5,6$ \\
\hline 12 & 5 & E II, R4, 5 \\
\hline 13 & 5 & E II, R4, 5, E VI > new $\mathrm{C}$ terminus \\
\hline 14 & 4 & R2-4 \\
\hline 15 & 4 & R4-6 \\
\hline 16 & 3 & R3-6 \\
\hline 17 & 1 & most of $\mathrm{R} 2$ to part of $E \mathrm{VI}$ * \\
\hline 18 & 1 & E II, most of R2 to part of E VI* \\
\hline
\end{tabular}

Source

\begin{tabular}{|c|c|}
\hline $\begin{array}{c}\text { Dendritic } \\
\text { cells }\end{array}$ & $\overline{\text { Rectum }}$ \\
\hline+ & \\
\hline+ & \\
\hline+ & \\
\hline+ & + \\
\hline & + \\
\hline+ & \\
\hline & $\begin{array}{l}+ \\
+\end{array}$ \\
\hline $\begin{array}{l}+ \\
+\end{array}$ & \\
\hline & + \\
\hline+ & $\begin{array}{l}+ \\
+\end{array}$ \\
\hline
\end{tabular}

d Soluble isoforms

$\begin{array}{cc}\text { Type } & \begin{array}{c}\text { No. of } \\ \text { repeats }\end{array} \\ \mathbf{1} & 7 \\ 2 & 6 \\ 3 & 6 \\ 4 & 5 \\ 5 & 5 \\ 6 & 3 \\ 7 & 2 \\ 8 & 2 \\ 9 & 1 \\ 10 & 0 \\ 11 & 0 \\ 12 & 0 \\ 13 & 0 \\ & \\ & \end{array}$

Source

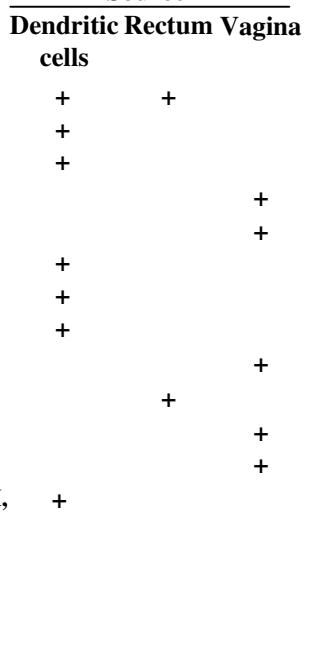

Figure 2 Predicted structure and molecular diversity of membrane-bound and soluble DC-SIGNR gene products. (a) and (b) Predicted structure of the prototypic membrane-bound (a) and soluble (b) DC-SIGNR gene product. Boxes are exons (E) I-VII, the numbered black boxes in exon IV denote the seven full $69 \mathrm{bp}$ repeats (R) and one partial repeat. Exons I, II, and a short portion of exon III encode the cytoplasmic domain. The transmembrane domain (TM) is encoded by the remaining sequence of exon III. The extracellular domain is composed of a short stretch of sequence just downstream of exon III, the seven full repeats and one half repeat of exon IV, and exons V, VI, and VII. Exons V, VI, and VII form the lectin-binding domain. Isoforms that lack the TM-encoding exon III are regarded as soluble. (c) Molecular diversity of membrane-bound DC-SIGNR gene products. In all, 18 types of mRNA transcripts containing exon III were found. Type 1 is the full-length transcript. (d) Molecular diversity of soluble DC-SIGNR gene products. In all, 13 types of mRNA transcripts lacking exon III were found. When exon VI $(113 \mathrm{bp})$ is spliced out, a frame shift occurs, which is predicted to lead to a novel C-terminus in the membrane-bound isoforms 3, 7, and 13, and in the soluble isoform 5 . In the membrane-bound isoforms 17 and 18 and the soluble isoforms 6 and $9-13$, the deletions cause premature stop codons (indicated by an asterisk). The lengths of all other deletions are multiples of three, causing no frame shift.

DC-SIGNR on isolated DC populations ${ }^{11,12,15}$ and the mucosa of vagina and rectum. ${ }^{5}$ This has been taken as evidence that DC-SIGNR is not involved in the pathogenesis of sexual HIV-1 transmission. Interestingly, however, we have found a correlation between the DC-SIGNR repeat region genotype and the susceptibility to HIV-1 infection, ${ }^{21}$ which does suggest a differential role of DC-SIGNR isoforms during viral transmission. This prompted us to explore the presence of DC-SIGNR in the vaginal and rectal mucosa with a more sensitive nested-PCR-based technique. $^{24,25}$ Using this strategy, we consistently detected DCSIGNR mRNA in all 13 vaginal and four rectal biopsies tested. The majority of these mRNAs were transcript isoforms resulting from genetic polymorphism of the repeat region in exon IV and alternative splicing. As indicated by cloning and sequencing of PCR products in three mucosal biopsies, an extensive transcript repertoire was generated locally, and its breadth was comparable to the repertoire detected in monocyte-derived DCs. Many of these transcript isoforms coded for proteins predicted to be soluble and secreted. ${ }^{16}$ Indeed, in one vaginal sample, we only found soluble isoforms.

Our studies suggest that transcripts coding for soluble isoforms are commonly expressed in the vaginal and rectal mucosa. We lack experimental evidence but presume that the DC-SIGNR and/or DC-SIGN mRNA transcripts missing the transmembrane anchoring region translate into proteins that are indeed secreted into the mucosal extracellular space. A recent study in vitro $^{28}$ supports this assumption.

The biological functions of the large repertoire of DCSIGNR and DC-SIGN isoforms remain unclear and speculations on this issue have been published by other authors. ${ }^{16,29}$ With respect to HIV transmission, our findings 


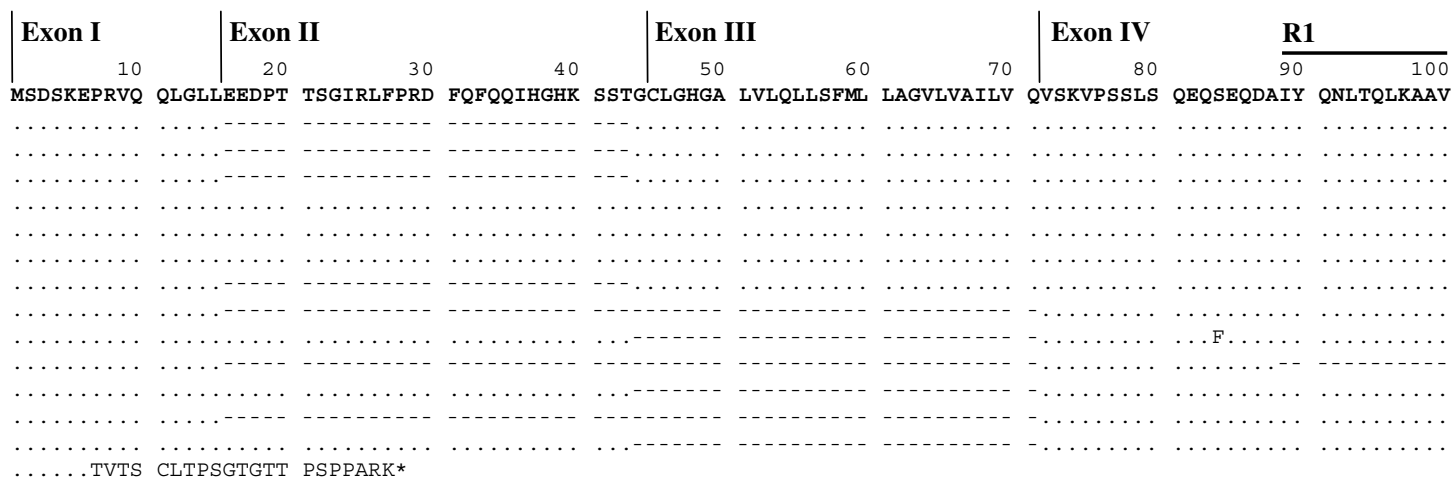

13 $\ldots \ldots$. . . . . . . ELAGL ELDPG*

$\underline{\mathrm{R} 2}$

$\underline{\mathrm{R} 3}$

$\frac{\mathbf{R} 4}{160}$

R5

$\begin{array}{ll}\text { m } & 1 \\ \text { m } & 2 \\ \text { m } & 3 \\ \text { m } & 4 \\ \text { m } & 6 \\ \text { m } & 1 \\ \text { m } & 1 \\ \text { m } & 1 \\ \text { s } & 1 \\ \text { s } & 2 \\ \text { s } & 3 \\ \text { s } & 5 \\ \text { s } & 7 \\ \text { s } & 9 \\ \text { s } & 1 \\ \text { s } & 1\end{array}$

GELSEKSKLO EIYOELTOLK AAVGELPEKS KLOEIYOELT RLKAAVGELP EKSKLOEIYO ELTRLKAAVG ELPEKSKLOE IYOELTRLKA AVGELPEKSK

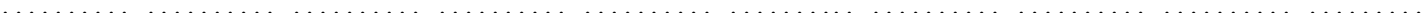

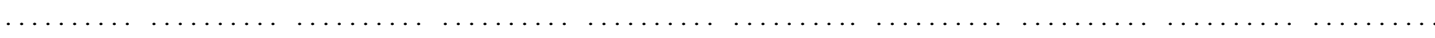

.

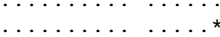

$\ldots \ldots \ldots \ldots+\cdots \cdots$

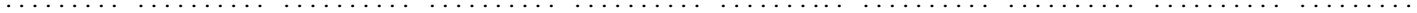

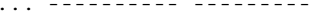

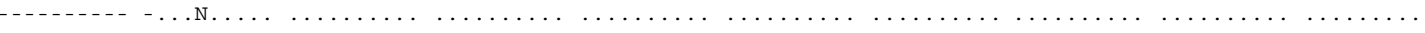

$\ldots \ldots \ldots$
$\ldots$. $\ldots \ldots$
$\ldots$

$\ldots \ldots \ldots \ldots \ldots$

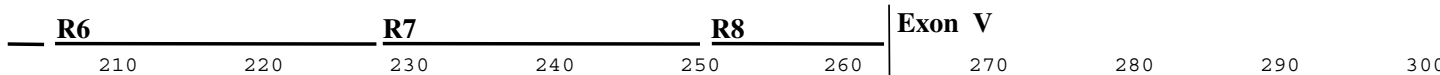

LQEIYQELTE LKAAVGELPE KSKLQEIYQE LTQLKAAVGE LPDQSKQQQI YQELTDLKTA FERLCRHCPK DWTFFQGNCY FMSNSQRNWH DSVTACQEVR

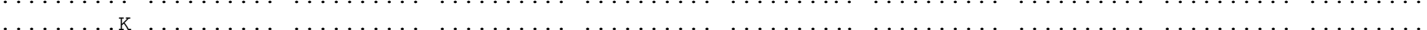

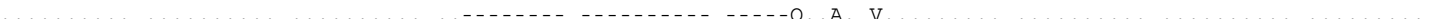

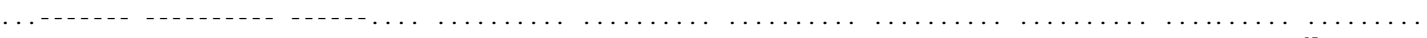

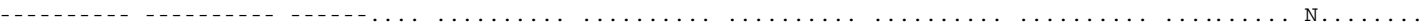

$\cdots$
$\cdots$

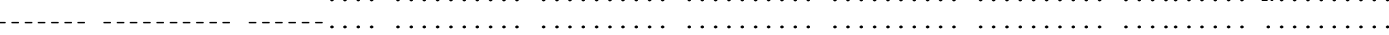

(1)

11

s 13

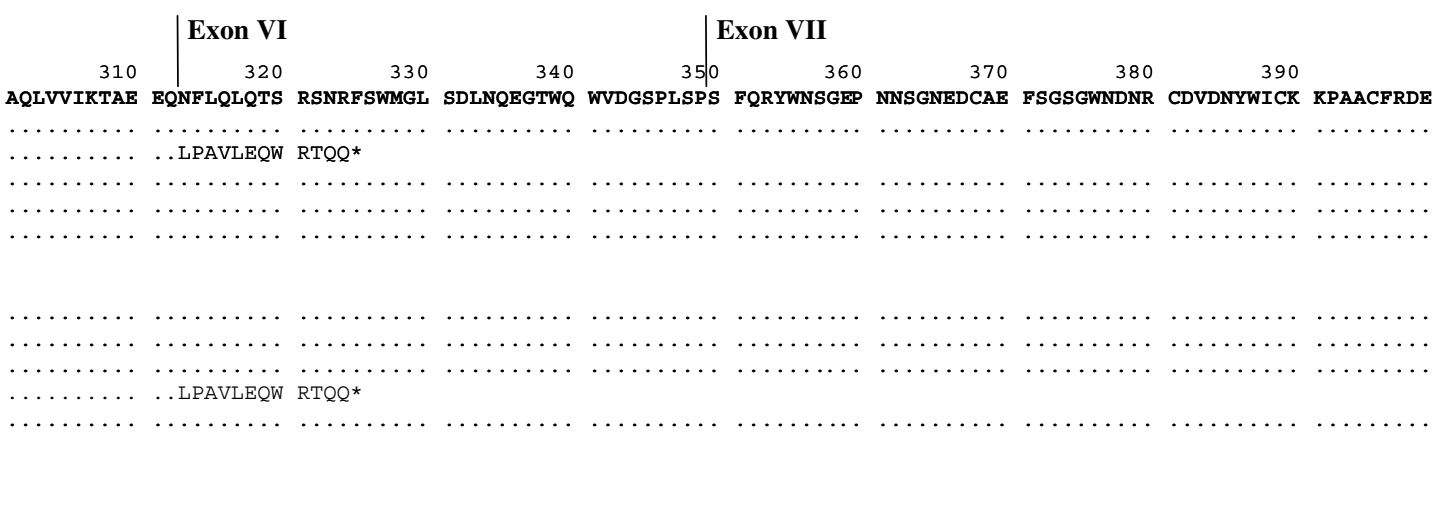

Figure 3 Alignment of predicted amino-acid sequences of representative DC-SIGNR isoforms. Membrane-bound DC-SIGNR isoforms are indicated by ' $\mathrm{m}$ ', soluble isoforms by ' $\mathrm{s}$ ', followed by the isoform type number as given in Figure 2 . The amino-acid sequence of $\mathrm{m} 1$ is the same as the deduced amino-acid sequence of the GenBank sequences AF209480S1 and AF209480S2. Dots indicate amino-acid identity to m1, dashes represent deletions, and stars indicate stop codons. The start of each of the 23 amino-acid repeats is denoted by the letter R (R1-R8) and always overlies isoleucine (I). 


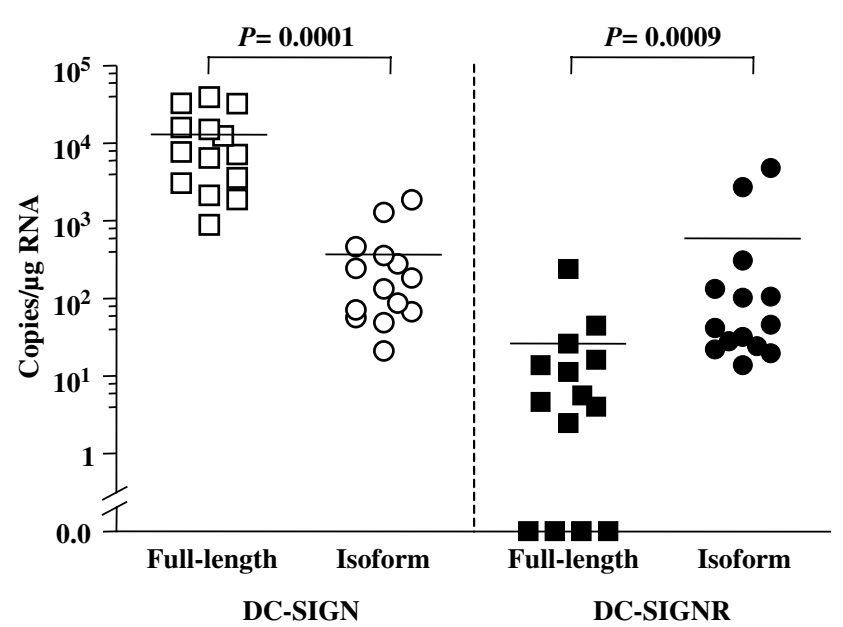

Figure 4 DC-SIGN and DC-SIGNR full-length and isoform mRNA copy numbers in vaginal and rectal biopsies. The mRNA copy numbers of full-length $(\square)$ and isoform $(\bigcirc)$ transcripts of DC-SIGN, and fulllength ( $\mathbf{0})$ and isoform (0) transcripts of DC-SIGNR are shown. Horizontal bars indicate the mean of each group. $P$-values were calculated using the Wilcoxon signed rank test.

in two separate studies that the DC-SIGNR and DC-SIGN repeat region genotypes affect HIV-1 infection, ${ }^{17,21}$ suggests that different isoforms may have distinct effects on HIV-1 pathogenesis. These differential effects may stem from variations in the relative concentrations of membrane-bound and soluble protein, and the capacity of isoforms to bind HIV-1 gp120. Since other investigators were unable to detect DC-SIGNR expression on mucosal cells by immunohistochemistry ${ }^{5}$ and, in the present study, we have observed much lower full-length than isoform mRNA copy numbers, we hypothesize that soluble isoforms are the most likely candidates to influence viral transmission. To address this, future studies must establish the presence of soluble DC-SIGNR protein in the tissue and determine the cellular source of DC-SIGNR in the mucosa, which most likely are endothelial ${ }^{11,12,14}$ or certain infiltrating DCs, as indicated by our and other studies. ${ }^{15,16}$ Most importantly, studies are required that compare the binding avidity for gp120 between DC-SIGNR isoforms generated from the 5-repeat allele and isoforms generated from the 7-repeat allele. Although the HIV-1 gp120 binding site of DC-SIGNR lies within the lectin-binding domain and not the repeat region, ${ }^{13,30}$ the repeat region is necessary for DC-SIGNR tetramerization. ${ }^{13,29}$ The simultaneous interaction of gp120 molecules with multiple subunits in a DC-SIGNR tetramer may be necessary for high avidity binding between DC-SIGNR and HIV-1. ${ }^{29,31}$ It is conceivable that the isoforms produced by specific repeat region alleles exhibit varying efficiencies of tetramerization and thus do not bind HIV-1 equally well. Our finding that the three individuals who lacked the 7-repeat allele failed to produce any full-length DC-SIGNR transcripts certainly suggests that the underlying genotype may influence the nature of the resulting transcript repertoire. Moreover, that out of a total of seven possible DC-SIGNR repeat region alleles, the 5- and the 7-repeat allele are by far the most frequent, ${ }^{11,21}$ points to these two alleles as the most likely candidates for differential regulatory functions.

In summary, we have demonstrated the presence of substantial amounts of DC-SIGNR and DC-SIGN isoform transcripts in the rectal and vaginal mucosa. We have also shown that an extensive repertoire of DC-SIGNR isoforms is generated locally, many of which code for proteins predicted to be soluble and secreted. ${ }^{16}$ In conjunction with our prior observation that the DC-SIGNR genotype is associated with HIV-1 susceptibility in vivo, ${ }^{21}$ these findings emphasize the need to further investigate the role of DCSIGNR and DC-SIGN protein products in mucosal immunity and HIV pathogenesis. In particular, it will be interesting to study the potential of soluble forms of these and other C-type lectins to function as antiviral compounds physiologically and therapeutically in vivo.

\section{Acknowledgements}

We thank EJ Soilleux for her suggestions concerning amplification of $D C$-SIGNR mRNA from DCs and her kind gift of DC-SIGNR CDNA, A Desbien for cell culture, C Wang for statistics, G Gao for helpful comments, $H$ Zhu, Y Hwangbo, and LM Hampson for technical assistance, PJ Nelson for patient recruitment, and M Bull for editing the manuscript. This work was supported by the National Institutes of Health Grants AI 45402 (TZ), AI 49109 (TZ), AI 56994 (TZ), AI 27757 (The University of Washington CFAR Clinical Research Core Award to HL, New Investigator Award to FH), AI 51980 (FH), AI 41535 (LC), AI 35605 (MJM), and a Burroughs Wellcome Clinical Scientist Award (MJM).

\section{References}

1 Geijtenbeek TB, Kwon DS, Torensma R et al: DC-SIGN, a dendritic cell-specific HIV-1-binding protein that enhances trans-infection of T cells. Cell 2000; 100: 587-597.

2 Sol-Foulon N, Moris A, Nobile C et al: HIV-1 Nef-induced upregulation of DC-SIGN in dendritic cells promotes lymphocyte clustering and viral spread. Immunity 2002; 16: 145-155.

3 Kwon DS, Gregorio G, Bitton N, Hendrickson WA, Littman DR: DC-SIGN-mediated internalization of HIV is required for transenhancement of T cell infection. Immunity 2002; 16: 135-144.

4 Pohlmann S, Baribaud F, Lee B et al: DC-SIGN interactions with human immunodeficiency virus type 1 and 2 and simian immunodeficiency virus. J Virol 2001; 75: 4664-4672.

5 Jameson B, Baribaud F, Pohlmann S et al: Expression of DC-SIGN by dendritic cells of intestinal and genital mucosae in humans and rhesus macaques. J Virol 2002; 76: 1866-1875.

6 Soilleux EJ, Morris LS, Leslie G et al: Constitutive and induced expression of DC-SIGN on dendritic cell and macrophage subpopulations in situ and in vitro. J Leukoc Biol 2002; 71: 445-457.

7 Sanders RW, de Jong EC, Baldwin CE, Schuitemaker JH, Kapsenberg ML, Berkhout B: Differential transmission of human immunodeficiency virus type 1 by distinct subsets of effector dendritic cells. J Virol 2002; 76: 7812-7821. 
8 Turville SG, Cameron PU, Handley A et al: Diversity of receptors binding HIV on dendritic cell subsets. Nat Immunol 2002; 3: 975-983.

9 Baribaud F, Pohlmann S, Leslie G, Mortari F, Doms RW: Quantitative expression and virus transmission analysis of DCSIGN on monocyte-derived dendritic cells. J Virol 2002; 76: 9135-9142.

10 Curtis B, Scharnowski S, Watson A: Sequence and expression of a membrane-associated C-type lectin that exhibits CD4independent binding of human immunodeficiency virus envelope glycoprotein gp120. Proc Natl Acad Sci USA 1992; 89: $8356-8360$

11 Bashirova AA, Geijtenbeek TBH, van Duijnhoven GCF et al: A Dendritic cell-specific intercellular adhesion molecule 3-grabbing nonintegrin (DC-SIGN)-related protein is highly expressed on human liver sinusoidal endothelial cells and promotes HIV-1 infection. J Exp Med 2001; 193: 671-678.

12 Pohlmann S, Soilleux EJ, Baribaud F et al: DC-SIGNR, a DC-SIGN homologue expressed in endothelial cells, binds to human and simian immunodeficiency viruses and activates infection in trans. Proc Natl Acad Sci USA 2001; 98: 2670-2675.

13 Feinberg H, Mitchell DA, Drickamer K, Weis WI: Structural basis for selective recognition of oligosaccharides by DC-SIGN and DCSIGNR. Science 2001; 294: 2163-2166.

14 Soilleux EJ, Morris LS, Rushbrook S, Lee B, Coleman N: Expression of human immunodeficiency virus (HIV)-binding lectin DCSIGNR: consequences for HIV infection and immunity. Hum Pathol 2002; 33: 652-659.

15 Soilleux EJ, Barten R, Trowsdale J: Cutting edge: DC-SIGN; a related gene, DC-SIGNR; and CD23 form a cluster on 19p13. J Immunol 2000; 165: 2937-2942.

16 Mummidi S, Catano G, Lam L et al: Extensive repertoire of membrane-bound and soluble DC-SIGN1 and DC- SIGN2 isoforms: inter-individual variation in expression of DC-SIGN transcripts. J Biol Chem 2001; 276: 33196-33212.

17 Liu H, Hwangbo Y, Holte S et al: Analysis of genetic polymorphisms in CCR5, CCR2, stromal cell-derived factor-1, RANTES, and dendritic cell-specific intercellular adhesion molecule-3-grabbing nonintegrin in seronegative individuals repeatedly exposed to HIV-1. J Infect Dis 2004; 190: 1055-1058.

18 Liu R, Paxton WA, Choe S et al: Homozygous defect in HIV-1 coreceptor status accounts for resistance of some multiplyexposed individuals to HIV-1 infection. Cell 1996; 86: 367-377.

19 Huang Y, Paxton WA, Wolinsky SM et al: The role of a mutant CCR5 allele in HIV-1 transmission and disease progression [see comments]. Nat Med 1996; 2: 1240-1243.
20 Dean M, Carrington M, Winkler $\mathrm{C}$ et al: Genetic restriction of HIV-1 infection and progression to AIDS by a deletion allele of the CKR5 structural gene. Hemophilia Growth and Development Study, Multicenter AIDS Cohort Study, Multicenter Hemophilia Cohort Study, San Francisco City Cohort, ALIVE Study. Science 1996; 273: 1856-1862.

21 Liu H, McElrath MJ, Holte $\mathrm{S}$ et al: Genetic polymorphisms in the DC-SIGNR repeat region affect HIV-1 transmission: 10th Conference on Retroviruses and Opportunistic Infections. Boston, 2003.

22 Romani N, Gruner S, Brang D et al: Proliferating dendritic cell progenitors in human blood. J Exp Med 1994; 180: 83-93.

23 Reddy A, Sapp M, Feldman M, Subklewe M, Bhardwaj N: A monocyte conditioned medium is more effective than defined cytokines in mediating the terminal maturation of human dendritic cells. Blood 1997; 90: 3640-3646.

$24 \mathrm{Zhu}$ T, Muthui D, Holte $\mathrm{S}$ et al: Evidence for human immunodeficiency virus type 1 replication in vivo in CD14(+) monocytes and its potential role as a source of virus in patients on highly active antiretroviral therapy. J Virol 2002; 76: 707-716.

25 Zhu T, Corey L, Hwangbo Y et al: Persistence of extraordinarily low levels of genetic homogeneous human immunodeficiency virus type 1 in exposed seronegative individuals. J Virol 2003; 77: $6108-6116$.

26 Rodrigo AG, Goracke PC, Rowhanian K, Mullins JI: Quantitation of target molecules from polymerase chain reaction-based limiting dilution assays. AIDS Res Hum Retroviruses 1997; 13: 737-742.

27 Kobayashi N, Nakamura HT, Goto $\mathrm{M}$ et al: Polymorphisms and haplotypes of the CD209L gene and their association with the clinical courses of HIV-positive Japanese patients. Jpn J Infect Dis 2002; 55: 131-133.

28 Butera S, Li J, Jia H, Blyveis N, Wilson C: Detection of a natural released form of soluble DC-SIGN in human dendritic cell cultures: 11th Conference on Retroviruses and Opportunistic Infections. San Francisco, 2004.

29 Pohlmann S, Baribaud F, Doms RW: DC-SIGN and DC-SIGNR: helping hands for HIV. Trends Immunol 2001; 22: 643-646.

30 Geijtenbeek TB, van Duijnhoven GC, van Vliet SJ et al: Identification of different binding sites in the dendritic cell-specific receptor DC-SIGN for intercellular adhesion molecule 3 and HIV-1. J Biol Chem 2002; 277: 11314-11320.

31 Mitchell DA, Fadden AJ, Drickamer K: A novel mechanism of carbohydrate recognition by the C-type lectins DC-SIGN and DCSIGNR. Subunit organization and binding to multivalent ligands. J Biol Chem 2001; 276: 28939-28945. 